\title{
Estimation of neutral lipid and carbohydrate quotas in microalgae using adaptive interval observers*
}

\author{
Francis Mairet ${ }^{1}$, Marcelo Moisan ${ }^{2}$, Olivier Bernard ${ }^{3}$ \\ 1: Departamentode Matemática, Universidad Técnica Federico Santa María, Avda \\ España 1680, Valparaíso, Chile \\ Email: francis.mairet@usm.cl \\ 2: EMEL S.A., BP 8330097, Santiago, Chile \\ 3: BIOCORE-INRIA, BP93, 06902 Sophia-Antipolis Cedex, France
}

\begin{abstract}
Under stress conditions, microalgae are known to accumulate large amounts of neutral lipids and carbohydrates, which can be used for biofuel production. However, on-line measurement of microalgal biochemical composition is a difficult task which makes the microalgal process rather difficult to manage. In this paper, we propose a so called adaptive interval observer for the on-line estimation of neutral lipid and carbohydrate quotas in microalgae. The observer is based on a change of coordinates that involves a time-varying gain. We introduce dynamics for the gain, whose trajectory converges toward a predefined optimal value (which maximizes the convergence rate of the observer). The observer performance is illustrated with experimental data of Isochrysis sp. cultures under nitrogen limitations and day-night cycle. The proposed observer design appears to be a suitable robust estimation technique.
\end{abstract}

Keywords: Adaptive interval observer, uncertain systems, microalgae biofuel, neutral lipid.

\section{Introduction}

Microalgae are a promising source of biomass for sustainable energy production [1]. Indeed, these photosynthetic micro-organisms can accumulate a large amount of neutral lipids (used for biodiesel production) and carbohydrates (used for methane or ethanol production by fermentation). However, given the current productivity, the large-scale industrial development of microalgal biofuel production is not yet foreseeable. One way to increase productivities is to develop monitoring and control strategies of microalgae culture [2]. However, on-line measurement of intracellular lipids is a difficult task which makes the microalgal process rather difficult to manage. As a first step, we propose an algorithm to estimate neutral lipid and carbohydrate quotas. Indeed, environmental conditions, such as light and nutrient availability, strongly affects microalgal growth but also its biochemical composition. Therefore, the estimation of neutral lipid and carbohydrate accumulations is a crucial tool for process control and optimization.

${ }^{*}$ Post-print - http://dx.doi.org/10.1007/s00449-013-0913-7 - Bioprocess and Biosystems Engineering, 2013. 
Given known bounds on all the uncertainties, interval observers [3, 4] allow to estimate the domain where the unmeasured variables are theoretically sure to be. This is a trending topic that has received increasing attention in the last decades. Based on the theory of positive differential systems [5], this robust state estimation technique is of particular interest for bioprocesses, which suffer from high uncertainties (on the inputs, measurements and model dynamics) and the lack of on-line measurements. Interval observers have been successfully applied to biological systems $[6,7,8,9]$, chaotic dynamics $[10,11]$, linear systems with additive disturbances [12], linear parameter-varying systems [13], etc. Assuming that a guaranteed bound of the initial unknown state and bounds on the uncertainties (inputs, disturbances, parameters, ...) are provided, a framer basically consists in an auxiliary dynamical system whose trajectories always stay above or below (component by component) those of the original system. Note that this definition does not impose any further constraint on the qualitative behavior of the framer. Therefore, an interval observer is a stable framer (with bounded error dynamics). One of the main advantages of interval observers is that the estimation performance can be evaluated online. Thanks to this property, several framers can be run in parallel $[6,14]$ (bundle of framers) and then the best estimates can be selected.

The main difficulty of this estimation approach is to define accurate bounds on the uncertainties: a too narrow estimate will not allow to guarantee the interval estimations of the unknown states, but large bounds will provide useless estimates. To tackle this drawback, new observer designs have been proposed recently in order to improve the estimation performance. In [7], an interval observer is designed based on a change of coordinates that involves a time-varying gain [15] which can be used to optimize the convergence rate of the estimation error. Nevertheless, this observer requires the gain derivative whose computation can be delicate (e.g. if the optimal value of the gain depends on the measurements). Thus, Mairet et al. [16] have introduced dynamics for the gain, whose trajectory converges toward a predefined optimal value (which maximizes the convergence rate of the observer). This estimation technique was called adaptive interval observer.

In this article, we propose the estimation of microalgal neutral lipid and carbohydrate quotas using adaptive interval observers. We first introduce a mathematical model of microalgal growth under light and nitrogen limitations. After recalling the concept of interval observer, we propose a design of interval observers for the estimation of neutral lipid and carbohydrate quotas. We then introduce the concept of adaptive interval observers and apply this concept to our problem. Finally, we validate the observer design with two sets of experimental data of Isochrysis sp. cultures.

\section{Model presentation}

Various models have been proposed to describe microalgae growth with respect to nutrient limitation and light (e.g. $[17,18,19])$. More recently, modelling microalgal neutral lipid production has also been tackled $[20,21,22]$. Our approach is based on the work developed in [20], which has been slightly modified in order to include light effect [23].

\subsection{The Droop model}

The modelling approach proposed in [20] is based on the Droop model, which was initially established to represent the effect of $\mathrm{B}_{12}$ vitamin internal quota on the growth rate of 
phytoplankton [24]. This model has been shown appropriate to represent also the effect of macronutrients, such as nitrogen, on growth rate [25, 26, 27]. Droop model considers that the growth of the biomass $x$ is related to the limited nutrient quota $q_{n}$, while nutrient uptake depends on the external concentration of nutrient $s$ (nitrate). Considering a perfectly mixed reactor, the Droop model reads:

$$
\left\{\begin{array}{l}
\dot{s}=D s_{i n}-\rho(s) x-D s \\
\dot{q_{n}}=\rho(s)-\mu\left(q_{n}\right) q_{n} \\
\dot{x}=\mu\left(q_{n}\right) x-D x
\end{array}\right.
$$

where $D$ is the dilution rate and $s_{i n}$ the influent nitrate concentration.

In this model the absorption rate $\rho(s)$ and growth rate $\mu\left(q_{n}\right)$ are generally taken as Michaelis-Menten and Droop functions:

$$
\begin{aligned}
& \rho(s)=\rho_{m} \frac{s}{s+K_{s}} \\
& \mu\left(q_{n}\right)=\mu_{m}\left(1-\frac{Q_{0}}{q_{n}}\right)
\end{aligned}
$$

where $K_{s}$ is the half saturation constant for substrate uptake and $Q_{0}$ the minimal cell quota. $\rho_{m}$ and $\mu_{m}$ are the maximum inorganic nitrogen uptake rate and the maximum growth rate, respectively.

\subsection{Lipid and carbohydrate dynamics}

Mairet et al. [20] have proposed a dynamical model describing the effects of nitrogen limitations on carbohydrate and neutral lipid accumulations under continuous light. This model considers a simplified carbon metabolism given in Figure 1. Carbon from $\mathrm{CO}_{2}$ is first incorporated as carbohydrates $g$. These carbohydrates are mobilized to produce functional carbon $f$ (mainly proteins) when microalgae uptake nitrogen. In parallel, carbohydrates are used to produce free fatty acids (FFA). These FFA can be stored as neutral lipid $l$ or mobilized to produce functional carbon (membranes). Considering this simplified metabolic network (see Fig. 1), the Droop model is completed by the dynamics of the neutral lipid $q_{l}$ and carbohydrate $q_{g}$ quotas [20]:

$$
\left\{\begin{array}{l}
\dot{q}_{l}=\left(\beta q_{n}-q_{l}\right) \mu\left(q_{n}\right)-\gamma \rho(s) \\
\dot{q}_{g}=\left(1-\beta q_{n}-q_{g}\right) \mu\left(q_{n}\right)-\alpha \rho(s)
\end{array}\right.
$$

The functional quota $q_{f}$ is given by $q_{f}=1-q_{l}-q_{g}$.

\subsection{Modelling the effect of light}

We propose to modify this model to deal with light variation. First, the light effect is represented through $\mu_{m}=\mu_{m}(I)[28]$ :

$$
\mu\left(q_{n}, I\right)=\mu_{m}(I)\left(1-\frac{Q_{0}}{q_{n}}\right)
$$

where we use for $\mu_{m}(I)$ the model of [29] taking into account photoinhibition:

$$
\mu_{m}(I)=\tilde{\mu}_{m} \frac{I}{I+K_{s I}+\frac{I^{2}}{K_{i I}}}
$$




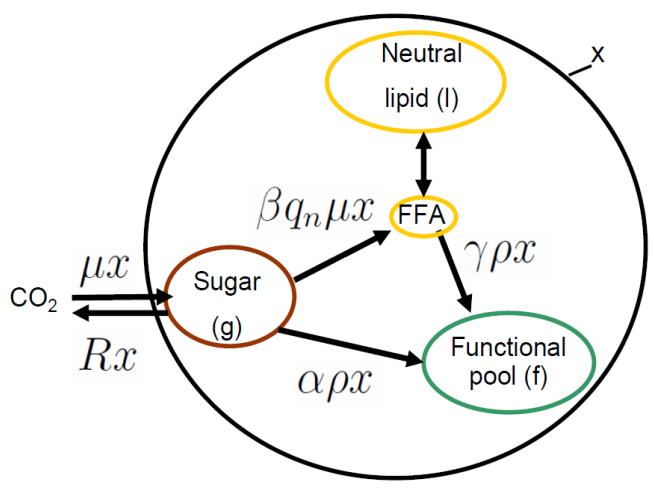

Figure 1: Representation of the intracellular carbon flows. Respiration has been added to the original scheme proposed by [20].

Parameter $K_{s I}$ refers to the half saturation coefficient with respect to light, $K_{i I}$ is an inhibition coefficient and $\tilde{\mu}_{m}$ defines the hypothetical maximal growth rate.

The nitrogen uptake rate must also be modified to deal with the light effect: nitrogen uptake is slower during the night [30] and it must stop when cells become replete (particularly in case of prolonged darkness):

$$
\rho\left(s, I, q_{n}\right)=\rho_{m} \frac{s}{s+K_{s}}\left(\eta+(1-\eta) \frac{I^{m}}{I^{m}+\epsilon_{I}^{m}}\right)\left(1-\frac{q_{n}}{Q_{l}}\right)
$$

where $Q_{l}$ is the maximal nitrogen quota, $\eta$ is the reduction factor of nitrogen uptake during the night, and $m$ a Hill coefficient.

Moreover, a respiration term is added. We assume that only carbohydrates are used for respiration. In line with [17], the respiration rate $R$ is taken as the sum of a constant maintenance respiration and a biosynthesis cost (proportional to the nitrogen uptake rate):

$$
R=r_{0}+r_{1} \rho\left(s, I, q_{n}\right)
$$

Note that the respiration was not included in [20] as the experimental validation was carried out under constant light. However, this term does not modify the behavior of the original model and its ability to describe the experimental data presented in [20].

Finally, we also add a nitrogen loss rate $r_{n}$.

\subsection{Resulting model}

Let us denote by $\chi=\left[s, q_{n}, x, q_{l}, q_{g}\right]^{T}$ the vector of model variables. Gathering all the elements presented in this paper, we obtain the following model:

$$
\left\{\begin{array}{l}
\dot{s}=D\left(s_{i n}-s\right)-\rho(\chi, I) x \\
\dot{q}_{n}=\rho(\chi, I)-\left[\mu(\chi, I)-R(\chi, I)+r_{n}\right] q_{n} \\
\dot{x}=[\mu(\chi, I)-D-R(\chi, I)] x \\
\dot{q}_{l}=\left(\beta q_{n}-q_{l}\right) \mu(\chi, I)-\gamma \rho(\chi, I)+R(\chi, I) q_{l} \\
\dot{q}_{g}=\left(1-\beta q_{n}-q_{g}\right) \mu(\chi, I)-\alpha \rho(\chi, I)-R(\chi, I)\left(1-q_{g}\right)
\end{array}\right.
$$


where $\mu(\chi, I)$ and $\rho(\chi, I)$ are given respectively by equations (4) and (6).

Note that this model can be used only for dilute culture. For high cell concentration, the light gradient inside the culture (due to microalgae self-shading) should be considered $[2,31]$.

\section{Interval observer design}

\subsection{Recall on interval observer}

We consider a differential system given by:

$$
(\Sigma):\left\{\begin{array}{l}
\dot{x}(t)=\mathrm{f}(x(t), u(t), \lambda) \\
y(t)=\mathrm{h}(x(t))
\end{array}\right.
$$

where $x(t)$ is the state variables, $u(t)$ is the inputs, $\lambda$ is a parameter vector, and $y$ is the measurement vector.

In the framework of parameter uncertainties, we assume that the real inputs and parameters are unknown, but that they can be enclosed by known quantities ${ }^{1}$ :

$$
\forall t \geq 0, \underline{u}(t) \leq u(t) \leq \bar{u}(t), \underline{\lambda} \leq \lambda \leq \bar{\lambda}
$$

A framer associated to the differential system $(\Sigma)$ is a differential system whose solutions generate guaranteed bounds for the state variables of $(\Sigma)[3]$.

Definition 1 A framer for System (9) where inputs and parameters satisfy Equation (10) is a pair of coupled dynamical systems:

$$
\left\{\begin{array}{l}
\dot{\bar{z}}=\bar{f}(\bar{z}, \underline{z}, \bar{u}(t), \underline{u}(t), \bar{\lambda}, \underline{\lambda}, \theta(t), y), \\
\underline{\dot{z}}=f(\bar{z}, \underline{z}, \bar{u}(t), \underline{u}(t), \bar{\lambda}, \underline{\lambda}, \theta(t), y), \\
\bar{x}=\overline{\bar{g}}(\bar{z}, \underline{z}, \bar{u}(t), \underline{u}(t), \bar{\lambda}, \underline{\lambda}, \theta(t), y), \\
\underline{x}=\underline{g}(\bar{z}, \underline{z}, \bar{u}(t), \underline{u}(t), \bar{\lambda}, \underline{\lambda}, \theta(t), y) \\
\underline{z}(0)=\underline{z}_{0} ; \bar{z}(0)=\bar{z}_{0},
\end{array}\right.
$$

such that we have $\forall t \geq 0$ :

$$
\underline{x}(t) \leq x(t) \leq \bar{x}(t)
$$

given $\underline{z}_{0}, \bar{z}_{0}$ such that $\underline{x}(0) \leq x(0) \leq \bar{x}(0)^{2}$.

Note that, in this design, we have introduced a time-varying parameter vector $\theta$ which can be used in order to tune the framer performance.

This definition is rather general, highlighting the fact that a framer is simply designed to give an upper and a lower bound of the unknown state. The following definition also includes stability properties.

Definition 2 A framer (11) with bounded predictions $\bar{x}$ and $\underline{x}$ is called an interval observer.

\footnotetext{
${ }^{1}$ All the inequalities must be understood component by component.

${ }^{2}$ In the following, this initialisation condition will always be assumed for all the framers.
} 


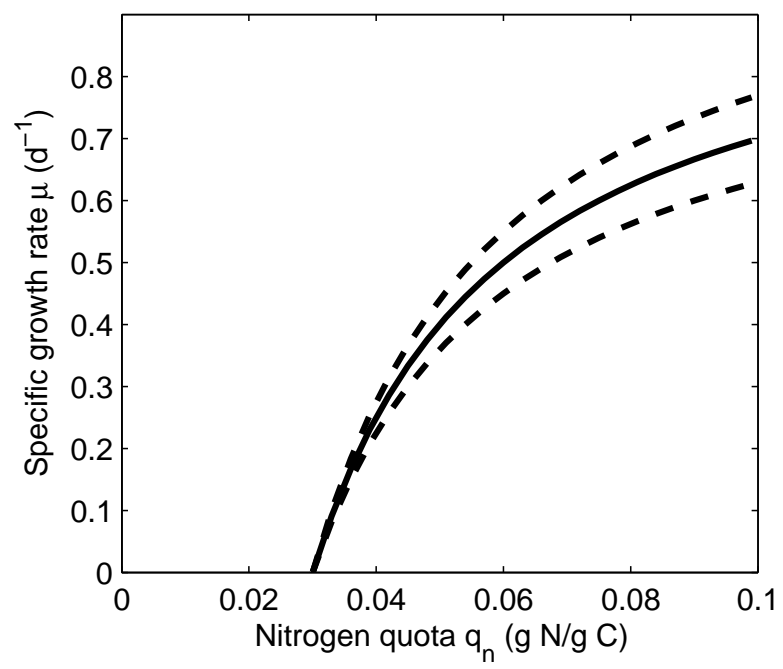

Figure 2: Bounds on the specific growth rate (see Hypothesis 1). Solid line: unknown function $\mu\left(q_{n}\right)$, dashed lines: known bounds $\underline{\mu}\left(q_{n}\right)$ and $\bar{\mu}\left(q_{n}\right)$.

\subsection{Framer design for microalgae}

Now we propose a framer design in order to estimate neutral lipid and carbohydrate quotas. For the sake of simplicity, the framer is first presented considering that $s, q_{n}$, and $x$ are perfectly measured and that the specific growth $(\mu)$ and the respiration $(R)$ rates are the only uncertain terms in System (8):

Hypothesis 1 (i) The specific growth rate is unknown but bounded by known functions $\underline{\mu}\left(q_{n}, I\right)$ and $\bar{\mu}\left(q_{n}, I\right)$ (see Fig. 2):

$$
\underline{\mu}\left(q_{n}, I\right) \leq \mu\left(q_{n}, I\right) \leq \bar{\mu}\left(q_{n}, I\right) .
$$

(ii) The respiration rate $R(\chi, I)$ is unknown but bounded by known parameters $\underline{R}$ and $\bar{R}$ :

$$
\underline{R} \leq R(\chi, I) \leq \bar{R} .
$$

Moreover, we consider that the nitrogen uptake rate $(\rho)$ is unknown. Thus, we introduce the following change of coordinates that is designed in order to eliminate the unknown uptake rate $\rho$ :

$$
\left\{\begin{array}{l}
z_{l}=\left(\theta_{l}-q_{l}\right) x+\gamma s \\
z_{g}=\left(1-\theta_{g}-q_{g}\right) x+\alpha s
\end{array}\right.
$$

where $\theta_{l}$ and $\theta_{g}$ are time-varying gains. The dynamics of $z_{l}$ and $z_{g}$ are given by:

$$
\left\{\begin{array}{l}
\dot{z}_{l}=D\left(\gamma s_{i n}-z_{l}\right)+\left(\theta_{l}-\beta q_{n}\right) \mu x-R x \theta_{l}+\dot{\theta}_{l} x \\
\dot{z}_{g}=D\left(\alpha s_{i n}-z_{g}\right)-\left(\theta_{g}-\beta q_{n}\right) \mu x+R x \theta_{g}-\dot{\theta}_{g} x
\end{array}\right.
$$

which let us introduce the following proposition.

Proposition 1 The following systems are framers of System (8):

- $f o r \theta_{i}^{+}(t) \geq \beta q_{n}, i=1, \ldots, 4$ 


$$
\begin{gathered}
\left\{\begin{array}{c}
\dot{\bar{z}}_{l}^{+}=D\left(\gamma s_{i n}-\bar{z}_{l}^{+}\right)-\underline{R} \theta_{1}^{+} x+\dot{\theta}_{1}^{+} x \\
+\left(\theta_{1}^{+}-\beta q_{n}\right) \bar{\mu}\left(q_{n}, I\right) x \\
\dot{\underline{z}}_{l}^{+}=D\left(\gamma s_{i n}-\underline{z}_{l}^{+}\right)-\bar{R} \theta_{2}^{+} x+\dot{\theta}_{2}^{+} x \\
\quad+\left(\theta_{2}^{+}-\beta q_{n}\right) \underline{\mu}\left(q_{n}, I\right) x \\
\bar{q}_{l}^{+}=\theta_{2}^{+}-\left(\underline{z}_{l}^{+}-\gamma s\right) / x \\
\underline{q}_{l}^{+}=\theta_{1}^{+}-\left(\bar{z}_{l}^{+}-\gamma s\right) / x
\end{array}\right. \\
\left\{\begin{array}{c}
\dot{\bar{z}}_{g}^{+}=D\left(\alpha s_{i n}-\bar{z}_{g}^{+}\right)+\bar{R} \theta_{3}^{+} x-\dot{\theta}_{3}^{+} x \\
-\left(\theta_{3}^{+}-\beta q_{n}\right) \underline{\mu}\left(q_{n}, I\right) x \\
\underline{\dot{z}}_{g}^{+}=D\left(\alpha s_{i n}-\underline{z}_{g}^{+}\right)+\underline{R} \theta_{4}^{+} x-\dot{\theta}_{4}^{+} x \\
-\left(\theta_{4}^{+}-\beta q_{n}\right) \bar{\mu}\left(q_{n}, I\right) x \\
\bar{q}_{g}^{+}=1-\theta_{4}^{+}-\left(\underline{z}_{g}^{+}-\alpha s\right) / x \\
\underline{q}^{+}=1-\theta_{3}^{+}-\left(\bar{z}_{g}^{+}-\alpha s\right) / x
\end{array}\right.
\end{gathered}
$$

- for $0 \leq \theta_{i}^{-}(t)<\beta q_{n}, i=1, \ldots, 4$

$$
\begin{gathered}
\left\{\begin{array}{c}
\dot{\bar{z}}_{l}^{-}=D\left(\gamma s_{i n}-\bar{z}_{l}^{-}\right)-\underline{R} \theta_{1}^{-} x+\dot{\theta}_{1}^{-} x \\
\quad+\left(\theta_{1}^{-}-\beta q_{n}\right) \underline{\mu}\left(q_{n}, I\right) x \\
\dot{\dot{z}}_{l}^{-}=D\left(\gamma s_{i n}-\underline{z}_{l}^{-}\right)-\bar{R} \theta_{2}^{-} x+\dot{\theta}_{2}^{-} x \\
\quad+\left(\theta_{2}^{-}-\beta q_{n}\right) \bar{\mu}\left(q_{n}, I\right) x \\
\bar{q}_{l}^{-}=\theta_{2}^{-}-\left(\underline{z}_{l}^{-}-\gamma s\right) / x \\
\underline{q}^{-}=\theta_{1}^{-}-\left(\bar{z}_{l}^{-}-\gamma s\right) / x
\end{array}\right. \\
\left\{\begin{array}{c}
\dot{\bar{z}}_{g}^{-}=D\left(\alpha s_{i n}-\bar{z}_{g}^{-}\right)+\bar{R} \theta_{3}^{-} x-\dot{\theta}_{3}^{-} x \\
-\left(\theta_{3}^{-}-\beta q_{n}\right) \bar{\mu}\left(q_{n}, I\right) x \\
\dot{z}_{g}^{-}=D\left(\alpha s_{i n}-\underline{z}_{g}^{-}\right)+\underline{R} \theta_{4}^{-} x-\dot{\theta}_{4}^{-} x \\
-\left(\theta_{4}^{-}-\beta q_{n}\right) \underline{\mu}\left(q_{n}, I\right) x \\
\bar{q}_{g}^{-}=1-\theta_{4}^{-}-\left(\underline{z}_{g}^{-}-\alpha s\right) / x \\
\underline{q}^{-}=1-\theta_{3}^{-}-\left(\bar{z}_{g}^{-}-\alpha s\right) / x
\end{array}\right.
\end{gathered}
$$

Proof Let us introduce the error $\bar{e}_{q_{l}}^{+}=\bar{q}_{l}^{+}-q_{l}$. Its dynamics read:

$$
\begin{aligned}
\dot{\bar{e}}_{q_{l}}^{+}=-\left[\mu\left(q_{n}, I\right)-\right. & R(\chi, I)] \bar{e}_{q_{l}}^{+}+\theta_{2}^{+}[R(\chi, I)-\underline{R}] \\
& +\left(\theta_{2}^{+}-\beta q_{n}\right)\left[\mu\left(q_{n}, I\right)-\underline{\mu}\left(q_{n}, I\right)\right]
\end{aligned}
$$

At time $t_{0}$, a correct initialisation of the framer gives $\bar{e}_{q_{l}}^{+}\left(t_{0}\right) \geq 0$. Now consider the time instant $t^{\star}$ such that $\bar{e}_{q_{l}}^{+}\left(t^{\star}\right)=0$, from Equation (18) we have $\dot{\bar{e}}_{q_{l}}^{+}\left(t^{\star}\right)>0$ and therefore the error will always stay positive, i.e. $\bar{q}_{l}^{+} \geq q_{l}, \forall t \geq t_{0}$. Similarly, one can easily check that all the errors (see in appendix the error dynamics) will stay positive, so the system (14)-(17) is a framer of system (8).

The time-variations of the gain $\theta_{i}^{+}$and $\theta_{i}^{-}$are arbitrary, and they can be chosen in order to optimize framer performances. We then take the inner envelope from the set of estimates generated by different gain values, that is:

$$
\left\{\begin{array}{l}
\overline{q_{l}}(t)=\min \left\{{\overline{q_{l}}}^{+}(t),{\overline{q_{l}}}^{-}(t)\right\} \\
\underline{q_{l}}(t)=\max \left\{\underline{q}^{+}(t), \underline{q_{l}}-(t)\right\} \\
\overline{q_{g}}(t)=\min \left\{\overline{q_{g}}+(t),{\overline{q_{g}}}^{-}(t)\right\} \\
\underline{q_{g}}(t)=\max \left\{\underline{q_{g}}+(t), \underline{q_{g}}-(t)\right\}
\end{array}\right.
$$


Note that it is not necessary to know the absorption rate $\rho(s)$ for implementing these framers.

\subsection{Optimal framers}

As it has been stated in [7], the optimal gains $\tilde{\theta}_{i}^{+}$and $\tilde{\theta}_{i}^{-}$minimizes the time derivative of the error at any time instant $t \geq t_{0}$, that is for $i=1, \ldots, 4$ :

$$
\begin{aligned}
& \tilde{\theta}_{i}^{+}(t)=\arg \min _{\theta_{i}^{+} \geq \beta q_{n}}\left\{J_{i}^{+}\left(\theta_{i}^{+}, \chi\right)\right\} \\
& \tilde{\theta}_{i}^{-}(t)=\arg \min _{0 \leq \theta_{i}^{-} \leq \beta q_{n}}\left\{J_{i}^{-}\left(\theta_{i}^{-}, \chi\right)\right\}
\end{aligned}
$$

with

$$
\left\{\begin{array} { l } 
{ J _ { 1 } ^ { + } ( \theta _ { 1 } ^ { + } , \chi ) = \dot { e } _ { q _ { l } } ^ { + } } \\
{ J _ { 2 } ^ { + } ( \theta _ { 2 } ^ { + } , \chi ) = \dot { \overline { e } } _ { q _ { l } } ^ { + } } \\
{ J _ { 3 } ^ { + } ( \theta _ { 3 } ^ { + } , \chi ) = \dot { e } _ { q _ { g } } ^ { + } } \\
{ J _ { 4 } ^ { + } ( \theta _ { 4 } ^ { + } , \chi ) = \dot { \overline { e } } _ { q _ { g } } ^ { + } }
\end{array} \quad \text { and } \quad \left\{\begin{array}{l}
J_{1}^{-}\left(\theta_{1}^{-}, \chi\right)=\dot{e}_{q_{l}}^{-} \\
J_{2}^{-}\left(\theta_{2}^{-}, \chi\right)=\dot{\bar{e}}_{q_{l}}^{-} \\
J_{3}^{-}\left(\theta_{3}^{-}, \chi\right)=\dot{e}_{q_{g}}^{-} \\
J_{4}^{-}\left(\theta_{4}^{-}, \chi\right)=\dot{\bar{e}}_{q_{g}}^{-}
\end{array}\right.\right.
$$

Given the error dynamics (see Equations (32) and (33)), it can be easily verified that the optimal gain value for $\theta_{i}^{+}$in $\left[\beta q_{n},+\infty\right)$ is $\tilde{\theta}^{+}=\beta q_{n}$. For $\theta_{i}^{-}$, the problem cannot be solved directly given that $\mu\left(q_{n}, I\right)$ and $R(\chi, I)$ are unknown. We should consider two cases:

- during the night: we have $\mu\left(q_{n}, 0\right)=0$, so the optimal gain is $\tilde{\theta}^{-}=0$,

- during the day, we assume that the errors on the growth rate $\bar{\mu}\left(q_{n}, I\right)-\mu\left(q_{n}, I\right)$ and $\mu\left(q_{n}, I\right)-\underline{\mu}\left(q_{n}, I\right)$ are greater than the errors on the respiration rate $\bar{R}(\chi, I)-R(\chi, I)$ and $R(\chi, \bar{I})-\underline{R}(\chi, I)$, so $\tilde{\theta}^{-}=\beta q_{n}$.

\section{Adaptive interval observers}

\subsection{Theoretical framework}

In this section, we present the concept of adaptive interval observer which has been proposed recently in [16]. We assume that there exists some optimal interval observer gain, noted $\tilde{\theta}(t)$, which optimizes some observer performance (e.g. the interval decreasing rate, see Section 3.3 or [7]). The constraint when using a time-varying parameter in the design of the framer is that its derivative $\dot{\theta}(t)$ must be used to compute $\bar{z}$ and $\underline{z}$. When dealing with an optimal value of this parameter, the computation of this derivative may reveal delicate since it may depend on the (unknown) state variable of system $(\Sigma)$ (see Section 3.3 or [7] for an example of the computation of this optimal parameter). Here we propose to use bounds on this optimal gain, and to introduce dynamics of adaptation so that the gain $\theta$ converges towards the optimal gain $\tilde{\theta}(t)$ :

$$
\dot{\theta}=\phi(\tilde{\theta}, \theta, \bar{\theta}, \underline{\theta})
$$

where adaptation dynamics of $\theta$ are driven by the mapping $\phi$. Functions $\bar{\theta}$ and $\underline{\theta}$ are bounds to ensure that $\theta$ stays in the parametric domain $\Theta$ for which System (11) is guaranteed to be a framer. Moreover, we assume that there exists a positive constant $\epsilon$ such that, for any time $t, \underline{\theta}(t)+\epsilon<\tilde{\theta}<\bar{\theta}(t)-\epsilon$. 
The mapping $\phi$ should be defined to guarantee that $\theta$ converges to $\tilde{\theta}$. In practice, this convergence must be fast compared to the original system (11) and a high gain strategy in the adaptation dynamics has therefore been chosen.

Since $\theta$ must be bounded between two known bounds $\underline{\theta}$ and $\bar{\theta}$ to satisfy sign conditions required for the framer, a possible choice of $\phi$ can be (for each component $\theta_{i}$ )

$$
\left\{\begin{array}{l}
\dot{\theta}_{i}=K_{i}^{a}\left(\tilde{\theta}_{i}-\theta_{i}\right)\left(1+\frac{\epsilon}{\overline{\theta_{i}}-\theta_{i}}+\frac{\epsilon}{\theta_{i}-\underline{\theta_{i}}}\right) \\
\left.\theta_{i}\left(t_{0}\right) \in\right] \underline{\theta}_{i}\left(t_{0}\right), \bar{\theta}_{i}\left(t_{0}\right)[
\end{array}\right.
$$

where $K_{i}^{a}$ are positive adaptation gains and $\epsilon$ a small constant.

For the sake of simplicity, since the dynamics of the observer gains $\theta_{i}$ are uncoupled, we will focus on one of the components and omit the subscript $i$ in the following. Thus, we will write generically $\theta$ instead of $\theta_{i}$.

Property 1 Assuming that the derivatives of $\underline{\theta}$ and $\bar{\theta}$ are bounded in norm by a constant $N$, and that there exists a positive constant $\epsilon$ such that $\underline{\theta}(t)+\epsilon<\tilde{\theta}<\bar{\theta}(t)-\epsilon$, then Equation (22) guarantees that for any $\theta\left(t_{0}\right)$ such that $\underline{\theta}\left(t_{0}\right)<\theta\left(t_{0}\right)<\bar{\theta}\left(t_{0}\right)$, we have $\underline{\theta}(t)<\theta(t)<\bar{\theta}(t), \forall t>t_{0}$.

Proof See [16].

In order to study the convergence of $\theta$ towards the optimal value $\tilde{\theta}$, let us define $\delta(t)=\theta(t)-\tilde{\theta}(t)$ whose dynamics is:

$$
\dot{\delta}=-K^{a} \delta\left(1+\frac{\epsilon}{\bar{\theta}-\tilde{\theta}-\delta}+\frac{\epsilon}{\delta+\tilde{\theta}-\underline{\theta}}\right)-\dot{\tilde{\theta}}
$$

Given Property 1 , we can restrict our analysis to the positively invariant (time-varying) set $\Delta=\{\delta \in \mathbb{R} \mid \underline{\theta}-\tilde{\theta}<\delta<\bar{\theta}-\tilde{\theta}\}$.

Property 2 Considering $\dot{\tilde{\theta}}$ as a (bounded) perturbation input, the system (23) in the invariant set $\Delta$ is input-to-state stable (ISS), i.e. there exists a class $\mathcal{K} \mathcal{L}$ function $\beta$ and a class $\mathcal{K}$ function $\gamma$ such that for any initial state $\delta\left(t_{0}\right) \in \Delta$ and any bounded input $\dot{\tilde{\theta}}(t)$, the solution $\delta(t)$ satisfies:

$$
\|\delta(t)\| \leq \beta\left(\left\|\delta\left(t_{0}\right)\right\|, t-t_{0}\right)+\gamma\left(\sup _{t_{0} \leq \tau \leq t}\|\dot{\tilde{\theta}}(\tau)\|\right)
$$

Moreover, the mapping $\gamma(r)=\frac{r}{(1-\alpha) K^{a}}$, (with $\left.0<\alpha<1\right)$ can be as small as desired on $\Delta$ by an appropriate choice of $K^{a}$.

Proof See [16].

Properties 1 and 2 ensure that $\theta$ will stay in the parametric domain $\Theta$ and will converge towards the optimal time-varying value $\tilde{\theta}$, and that the ultimate bound can be as small as desired by the choice of a large gain $K^{a}$. In particular, from Property $2, \delta(t)$ remains bounded for bounded input $\dot{\tilde{\theta}}$, with an ultimate bound which is a function of the input magnitude. Moreover, if the optimal gain $\tilde{\theta}$ is constant, then system (23) is globally asymptotically stable. In practice, $\epsilon$ can be chosen small enough such that the two rational functions of Equations (22) affect the dynamic only if $\theta$ is very close to a bound. 


\subsection{Application to microalgae (continued)}

The implementation of the optimal framer defined by Equations (14)-(17) with $\theta=\beta q_{n}$ requires derivative computation of $q_{n}$, or at least bounded estimations, which are difficult to provide accurately. Therefore, in application of the main idea presented in Section 4.1, the framer is modified introducing an adaptive dynamics for $\theta$ :

Proposition 2 A near-optimal framer for System (8) is given by System (14)-(17) with the gain dynamics:

$$
\left\{\begin{array}{l}
\dot{\theta}_{i}^{+}=\phi\left(\beta q_{n}+\epsilon, \theta_{i}^{+},+\infty, \beta q_{n}\right) \\
\dot{\theta}_{i}^{-}=\phi\left(\tilde{\theta}^{-}(t), \theta_{i}^{-}, \beta q_{n}, 0\right)
\end{array}\right.
$$

where the mapping $\phi$ is defined by Equation (22), and

$$
\tilde{\theta}^{-}(t)=\left\{\begin{array}{l}
\epsilon \text { if } I(t)=0 \\
\beta q_{n}-\epsilon \text { if } I(t)>0
\end{array}\right.
$$

Proof From Property 1, the mapping $\phi$ ensures that the gains $\theta_{i}^{+}(t)$ and $\theta_{i}^{-}(t)$ stay in the parametric domain for which System (14)-(17) is a framer. Therefore, in direct consequence of Proposition 1, this system is a framer for System (8), with a near-optimal convergence of the error given that the gains $\theta_{i}^{+}(t)$ and $\theta_{i}^{-}(t)$ will converge towards the optimal values defined in Section 3.3 (see Proposition 2).

Note that, in this case, the optimal gains should be chosen as close as possible to the bounds. Nevertheless, to satisfy the hypotheses of Property 1, we select the near optimal gain: $\tilde{\theta}=\beta q_{n} \pm \epsilon$ or $\tilde{\theta}=\epsilon$, for a small $\epsilon$.

\section{Validation with experimental data}

\subsection{Including the uncertainties on parameters and the measurement noise}

In order to validate our approach with experimental data, we should consider a more realistic framework where all model parameters suffer from uncertainties and only $s$ and $x$ are measured, with noise perturbation. First, we provide an interval estimation of $q_{n}$, and then, a new framer for System (8) is proposed using the estimation of $q_{n}$ in the gain dynamics.

Hypothesis 2 Online measurements $y_{s}(t)$ and $y_{x}(t)$ are perturbed by noises $\delta_{s}(t)$ and $\delta_{x}(t)$. We assume that these perturbations are of multiplicative nature:

$$
y_{s}(t)=s(t)\left(1+\delta_{s}(t)\right) \quad \text { and } \quad y_{x}(t)=x(t)\left(1+\delta_{x}(t)\right)
$$

Moreover, these noise signals are bounded such that $\left|\delta_{s}(t)\right| \leq \Delta_{s}<1$ and $\left|\delta_{x}(t)\right| \leq$ $\Delta_{x}<1$.

We can define dynamic bounds for the substrate and the biomass:

$$
\underline{y}_{s}(t) \leq s(t) \leq \bar{y}_{s}(t) \quad \text { and } \quad \underline{y}_{x}(t) \leq x(t) \leq \bar{y}_{x}(t)
$$

with

$$
\left\{\begin{array} { l } 
{ y _ { s } ( t ) = \frac { y _ { s } ( t ) } { ( 1 + \Delta _ { s } ) } } \\
{ \overline { y } _ { s } ( t ) = \frac { y _ { s } ( t ) } { ( 1 - \Delta _ { s } ) } }
\end{array} \quad \text { and } \quad \left\{\begin{array}{l}
y_{x}(t)=\frac{y_{x}(t)}{\left(1+\Delta_{x}\right)} \\
\bar{y}_{x}(t)=\frac{y_{x}(t)}{\left(1-\Delta_{x}\right)}
\end{array}\right.\right.
$$



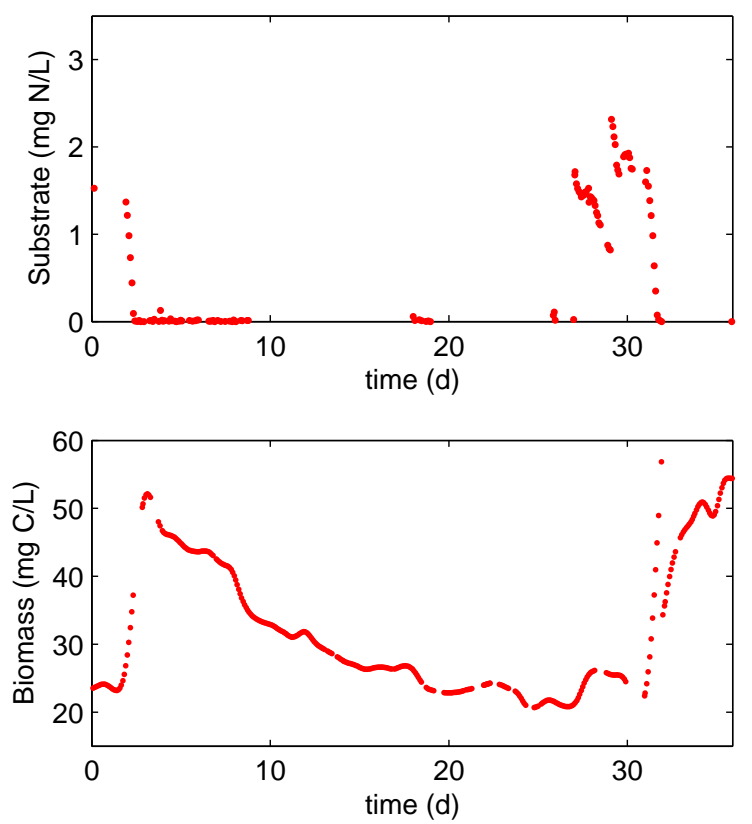

Figure 3: First experiment (nitrogen limitations). Substrate (top) and biomass measurements (down) [32].

\subsubsection{Interval estimation of $q_{n}$}

An interval estimator of the nitrogen quota $q_{n}$ is designed using a change of variable to eliminate the reaction rates $\rho(s)$ and $\mu(q)$

$$
\zeta=s+q_{n} x
$$

whose dynamics is $\dot{\zeta}=D\left(s_{i n}-\zeta\right)-r_{n}(\zeta-s)$.

Property 3 Given $\underline{s}_{i n}$ and $\overline{s_{i n}}$ such that $s_{i n} \in\left[s_{i n} ; \overline{s_{i n}}\right]$, and $\underline{r}_{n}$ and $\bar{r}_{n}$ such that $r_{n} \in$ $\left[\underline{r}_{n} ; \bar{r}_{n}\right]$, the following framer will provide bounds for the nitrogen quota $q_{n}$ :

$$
\left\{\begin{array}{l}
\dot{\bar{\zeta}}=D\left(\overline{s_{i n}}-\bar{\zeta}\right)-\underline{r}_{n}(\bar{\zeta}-s) \\
\dot{\zeta}=D\left(\underline{s}_{i n}-\bar{\zeta}\right)-\bar{r}_{n}(\underline{\zeta}-s) \\
\overline{\bar{q}}_{n}=\left(\bar{\zeta}_{y}\right) / \underline{y}_{x} \\
\underline{q}_{n}=\left(\underline{\zeta}-\bar{y}_{s}\right) / \bar{y}_{x}
\end{array}\right.
$$

Proof Computing the dynamics of the errors $\bar{e}_{\zeta}=\bar{\zeta}-\zeta$ and $\underline{e}_{\zeta}=\zeta-\zeta$, it is straightforward to show that they stay positive after a positive initialization. Then, we have:

$$
\begin{aligned}
& \bar{q}_{n}=\left(\bar{\zeta}-\underline{y}_{s}\right) / y_{x} \geq\left(\zeta-y_{s}\right) / y_{x}=q_{n} \\
& \underline{q}_{n}=\left(\underline{\zeta}-\bar{y}_{s}\right) / \bar{y}_{x} \leq\left(\zeta-y_{s}\right) / y_{x}=q_{n}
\end{aligned}
$$

which concludes the proof. 


\subsubsection{Interval estimation of $q_{l}$}

The framer given in Proposition 2 has been modified in order to take into account all the uncertainties:

Property 4 The following systems are framers of System (8):

- $f \circ \theta^{+}(t) \geq \bar{\beta} \overline{q_{n}}$

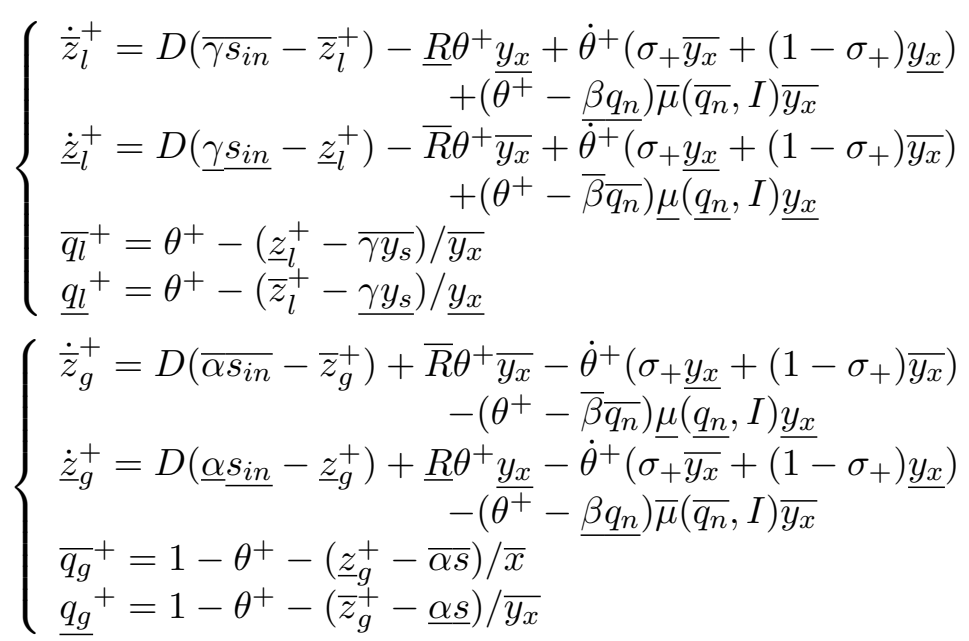

- for $0 \leq \theta^{-}(t)<\underline{\beta q_{n}}$

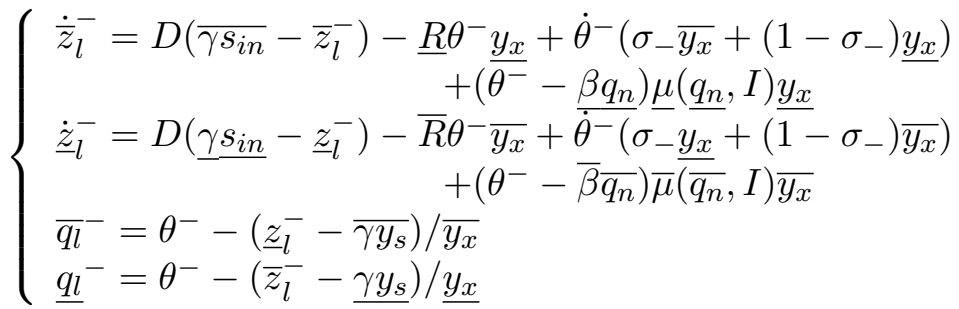

$$
\begin{aligned}
& \left\{\begin{array}{c}
\dot{\bar{z}}_{g}^{-}=D\left(\overline{\alpha s_{i n}}-\bar{z}_{g}^{-}\right)+\bar{R} \theta^{-} \overline{y_{x}}-\dot{\theta}^{-}\left(\sigma_{-} y_{x}+\left(1-\sigma_{-}\right) \overline{y_{x}}\right) \\
\quad-\left(\theta^{-}-\bar{\beta} \overline{q_{n}}\right) \bar{\mu}\left(\overline{q_{n}}, I\right) \overline{y_{x}} \\
\underline{\dot{z}}_{g}^{-}=D\left(\underline{\alpha} \underline{s_{i n}}-\underline{z}_{g}^{-}\right)+\underline{R} \theta^{-}-\left(\overline{y x}_{\theta^{-}}-\dot{\theta}^{-}\left(\sigma_{-} \overline{y_{x}}+\left(1-\sigma_{-}\right) \underline{y_{n}}\right)\right. \\
\underline{\mu}\left(\underline{q_{n}}, I\right) \underline{y_{x}} \\
{\overline{q_{g}}}^{-}=1-\theta^{-}-\left(\underline{z}_{g}^{-}-\overline{\alpha s}\right) / \bar{x} \\
{\underline{q_{g}}}^{-}=1-\theta^{-}-\left(\bar{z}_{g}^{-}-\underline{\alpha s}\right) / \overline{y_{x}}
\end{array}\right.
\end{aligned}
$$

where $q_{n}$ and $\overline{q_{n}}$ are given by Framer (25),

$\sigma_{ \pm}=\left\{\begin{array}{ll}1 & \text { if } \dot{\theta}^{ \pm} \geq 0 \\ 0 & \text { otherwise }\end{array}\right.$, uses the sign of $\dot{\theta^{ \pm}}$in order to provide the correct bounding, and the gain dynamics are:

$$
\left\{\begin{array}{l}
\dot{\theta}^{+}=\phi\left(\bar{\beta} \overline{q_{n}}+\epsilon, \theta^{+},+\infty, \bar{\beta} \overline{q_{n}}\right) \\
\dot{\theta}^{-}=\phi\left(\tilde{\theta}^{-}(t), \theta^{-}, \underline{\beta q_{n}}, 0\right)
\end{array}\right.
$$

where the mapping $\phi$ is defined by Equation (22).

Proof As for the previous framers, after the computation of the error dynamics, one can show that they stay positive after a positive initialization. 


\subsection{Experimental validation}

Table 1: Model parameters used for the experimental validation

\begin{tabular}{lcc}
\hline Parameter & $\begin{array}{c}\text { First } \\
\text { experiment }\end{array}$ & $\begin{array}{c}\text { Second } \\
\text { experiment }\end{array}$ \\
\hline$Q_{0}\left(g[N] \cdot g[C]^{-1}\right)$ & 0.05 & 0.046 \\
$\tilde{\mu}_{m}\left(d^{-1}\right)$ & 2.11 & 7.8 \\
$K_{s I}\left(\mu m o l . m^{-2} \cdot s^{-1}\right)$ & - & 330 \\
$\alpha\left(g[C] . g[N]^{-1}\right)$ & 2.6 & 3 \\
$\beta\left(g[C] \cdot g[N]^{-1}\right)$ & 4.8 & 3.8 \\
$\gamma\left(g[C] \cdot g[N]^{-1}\right)$ & 3.0 & 2.9 \\
$R\left(d^{-1}\right)$ & - & 0.05 \\
$r_{n}\left(d^{-1}\right)$ & - & 0.01 \\
\hline
\end{tabular}

The framer performances are assessed with two sets of experimental data of Isochrysis sp. (clone T-iso) continuous cultures:

- The first experiment consists in imposing various nitrogen limitations through a succession of dilution rate changes under constant light.

- The second experiment consists in a non-limited growth followed by a nitrogen starvation under day-night cycle.

More details about the experiments and operating conditions can be found in $[32,33,20$, 23]. Model parameter values are given in Table 1 . We consider a $\pm 10 \%$ uncertainty for the maximum specific growth rate $\tilde{\mu}_{m}$, a $\pm 2 \%$ uncertainty for the other model parameters and a multiplicative noise on the measurements up to a $2 \%$. We take for adaptation gain value $K^{a}=10$. Figures 3 and 5 present the substrate and biomass measurements $y_{s}(t)$ and $y_{x}(t)$ which are used to estimate the nitrogen and lipid quotas. The framer (27)-(30) provides an accurate interval estimation of the nitrogen, neutral lipid and carbohydrate quotas (see Figures 4 and 6) where almost all the measurements lie. Note that a few measurements are outside the interval estimation. This can be due to a narrow estimate of uncertainties (on parameters and measurements), or also to measurement errors. For the first experiment, we can observe perturbations at days 27 and 29 due to nitrate feeding impulses. After that, the framer (27)-(30) rapidly provides an accurate estimation. The interval estimation for the second experiment is less accurate given the additional effect of light which introduces more uncertainties. Note that, on Fig. 7, we can check that the tuning parameters $\theta^{+}$and $\theta^{-}$rapidly converge toward their optimal value while remaining within the bounds.

\section{Conclusions}

Analytical measurement of lipids in microalgae, and especially the determination of neutral and polar quotas are difficult and cannot be carried out on-line. Here we propose an interval observer using measurements of biomass and residual nitrate. After estimation of the optimal gain, this estimation algorithm turns out to be accurate enough to support a supervision approach allowing on-line process optimisation. The proposed framework is rather straightforward which makes its on-line implementation simple, provided that 

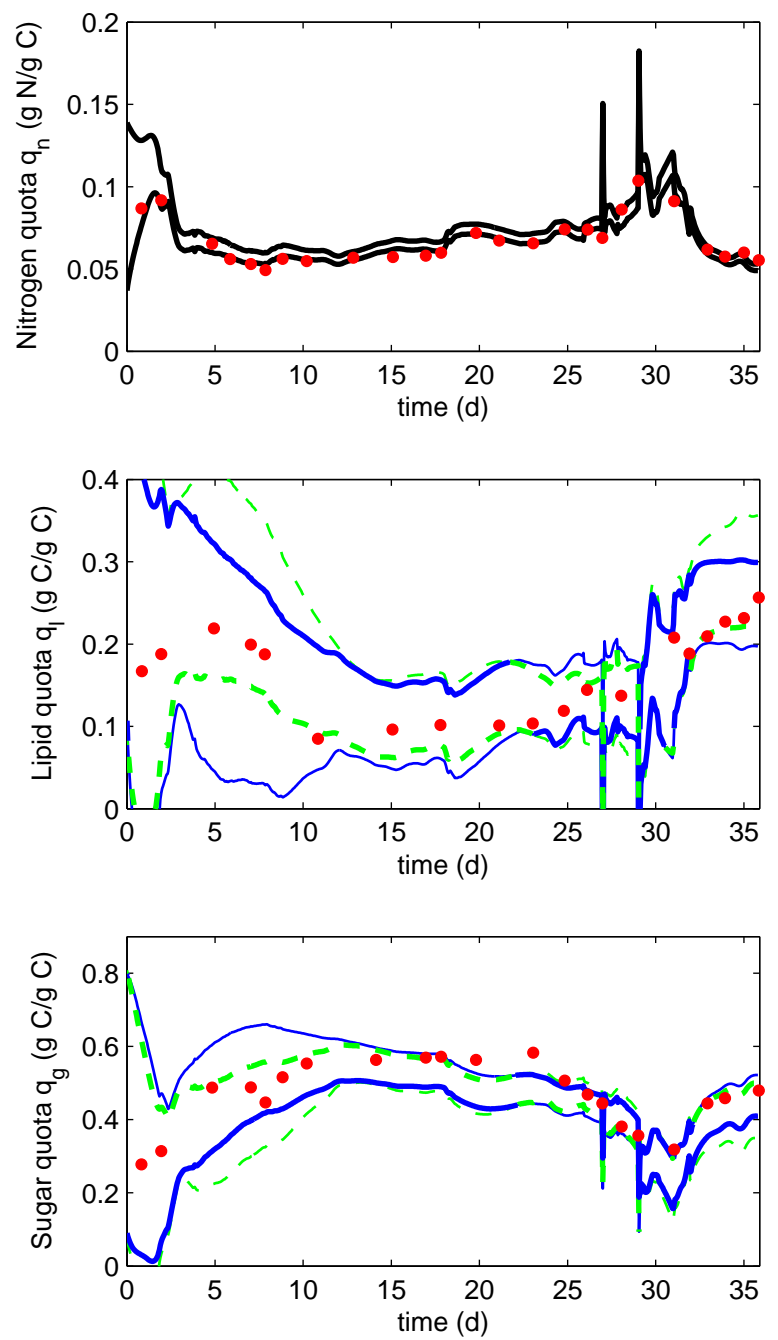

Figure 4: First experiment (nitrogen limitations). Interval estimations of the nitrogen (top), neutral lipid (middle) and carbohydrate (down) quotas provided by Framer (27)(30). Blue lines: estimation with the gain $\theta^{+}$, green dashed lines: estimation with the gain $\theta^{-}$, thick lines: the inner envelope. Red dots: experimental data for validation of the interval estimation. 

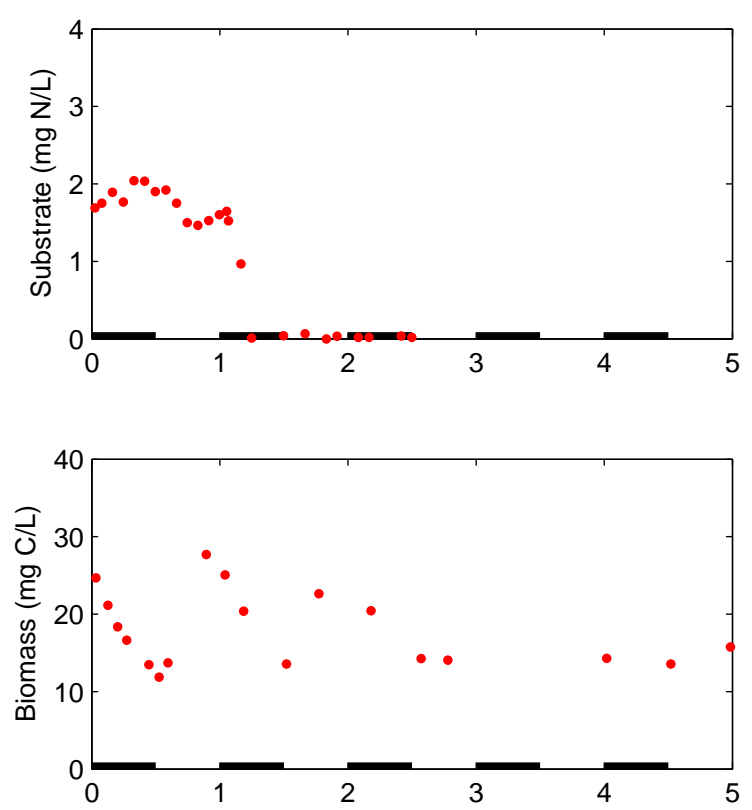

Figure 5: Second experiment (day-night cycle). Substrate (top) and biomass measurements (down) [32]. The black bars on the time axis represent the night periods.

an on-line measurement of biomass and remaining extracellular nitrate are available. It should now be associated to a calibration procedure to make its use more easy for other species.

\section{$7 \quad$ Appendix}

Computation of the error dynamics for Framer (14)-(17). Let us introduce the errors

$$
\left\{\begin{array} { l } 
{ \overline { e } _ { q _ { l } } ^ { + } = \overline { q } _ { l } ^ { + } - q _ { l } } \\
{ \underline { e } _ { q _ { l } } ^ { + } = q _ { l } - \underline { q l } ^ { + } } \\
{ \overline { e } _ { q _ { l } } ^ { + } = \overline { q } _ { g } + \underline { - } q _ { g } } \\
{ \underline { e } _ { q _ { g } } ^ { + } = q _ { g } - \underline { q } ^ { + } }
\end{array} \quad \text { and } \quad \left\{\begin{array}{l}
\bar{e}_{q_{l}}^{-}=\bar{q}_{l}^{-}-q_{l} \\
\underline{e}_{q_{l}}^{-}=q_{l}-\underline{q_{l}} \\
\bar{e}_{q_{l}}^{-}=\overline{q_{g}}-\underline{-} q_{g} \\
\underline{e}_{q_{g}}^{-}=q_{g}-\underline{q}^{-}
\end{array}\right.\right.
$$

Their dynamics read:

$$
\left\{\begin{array}{c}
\dot{\bar{e}}_{q_{l}}^{+}=-G \bar{e}_{q_{l}}^{+}+\left(\theta_{2}^{+}-\beta q_{n}\right) \underline{e}_{\mu}+\theta_{2}^{+} \bar{e}_{R} \\
\dot{e}_{q_{l}}^{+}=-G \underline{e}_{q_{l}}^{+}+\left(\theta_{1}^{+}-\beta q_{n}\right) \bar{e}_{\mu}+\theta_{1}^{+} \underline{e}_{R} \\
\dot{\bar{e}}_{q_{g}}^{+}=-G \bar{e}_{q_{g}}^{+}+\left(\theta_{4}^{+}-\beta q_{n}\right) \bar{e}_{\mu}+\theta_{4}^{+} \underline{e}_{R} \\
\dot{e}_{q_{g}}^{+}=-G \underline{e}_{q_{g}}^{+}+\left(\theta_{3}^{+}-\beta q_{n}\right) \underline{e}_{\mu}+\theta_{3}^{+} \bar{e}_{R}
\end{array}\right.
$$

and

$$
\left\{\begin{array}{l}
\dot{\bar{e}}_{q_{l}}^{-}=-G \bar{e}_{q_{l}}^{-}-\left(\theta_{2}^{-}-\beta q_{n}\right) \bar{e}_{\mu}+\theta_{2}^{-} \bar{e}_{R} \\
\dot{e}_{q_{l}}^{-}=-G \underline{e}_{q_{l}}^{-}-\left(\theta_{1}^{-}-\beta q_{n}\right) \underline{e}_{\mu}+\theta_{1}^{-} \underline{e}_{R} \\
\dot{\bar{e}}_{q_{g}}^{-}=-G \bar{e}_{q_{g}}^{-}-\left(\theta_{4}^{-}-\beta q_{n}\right) \underline{e}_{\mu}+\theta_{4}^{-} \underline{e}_{R} \\
\dot{e}_{q_{g}}^{-}=-G \underline{e}_{q_{g}}^{-}-\left(\theta_{3}^{-}-\beta q_{n}\right) \bar{e}_{\mu}+\theta_{3}^{-} \bar{e}_{R}
\end{array}\right.
$$



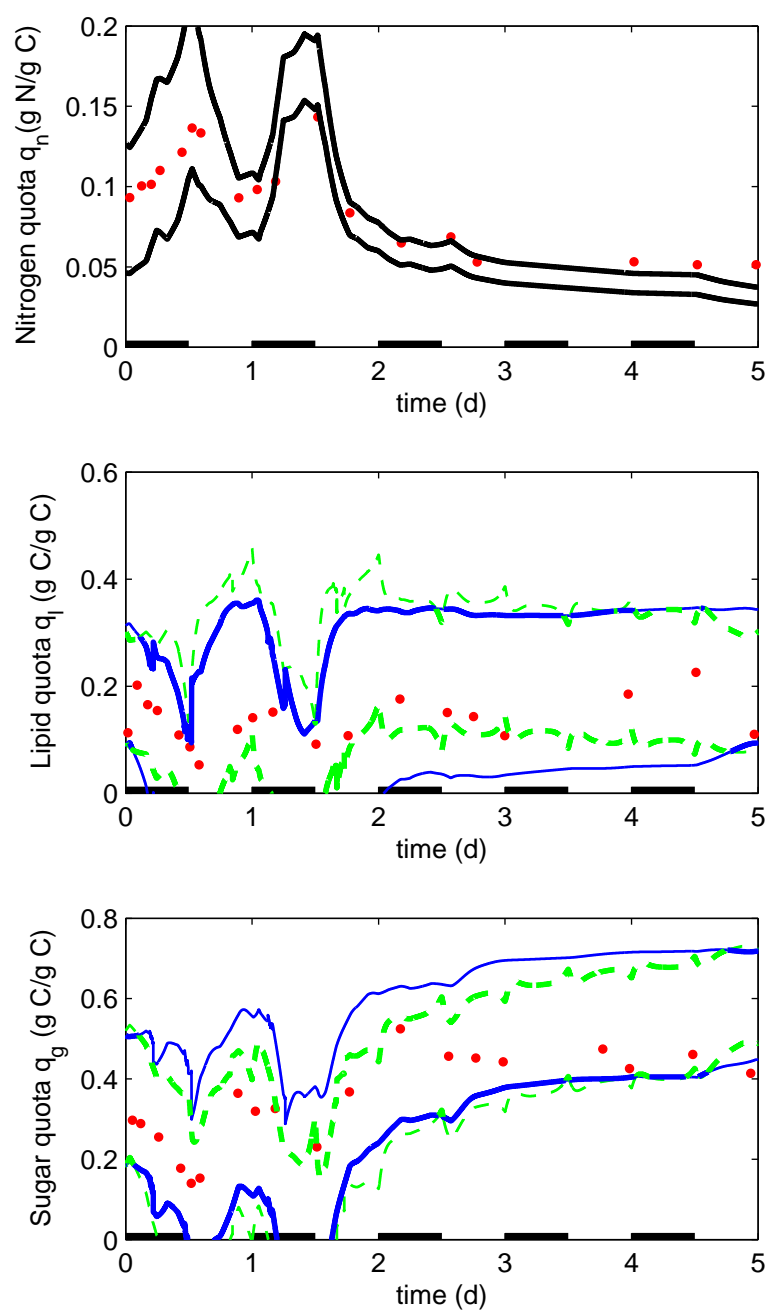

Figure 6: Second experiment (day-night cycle). Interval estimations of the nitrogen (top), neutral lipid (middle) and carbohydrate (down) quotas provided by framer (27)-(30). Blue lines: estimation with the gain $\theta^{+}$, green dashed lines: estimation with the gain $\theta^{-}$, thick lines: the inner envelope. Red dots: experimental data for validation of the interval estimation. 


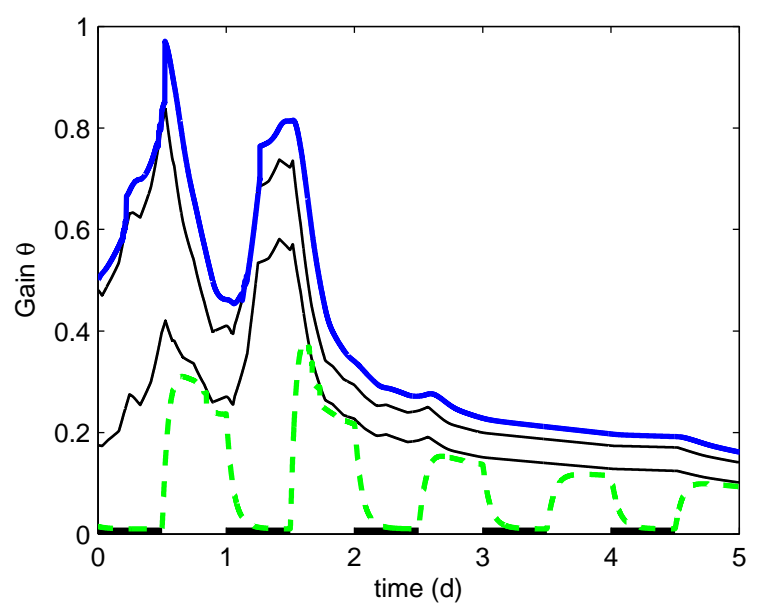

Figure 7: Evolution of the tuning parameters $\theta$. Thin black lines: $\overline{\beta q_{n}}$ and $\beta q_{n}$, thick lines: tuning parameters $\theta^{+}$(in blue) and $\theta^{-}$(in dashed green). $\theta^{+}$tracks $\overline{\beta q_{n}}$ while $\theta^{-}$ tracks $\underline{\beta q_{n}}$ during the day and it tends to zero during the night.

with:

$$
\left\{\begin{array}{l}
G=\mu\left(q_{n}, I\right)-R(\chi, I) \\
\bar{e}_{\mu}=\bar{\mu}\left(q_{n}, I\right)-\mu\left(q_{n}, I\right) \\
\underline{e}_{\mu}=\mu\left(q_{n}, I\right)-\mu\left(q_{n}, I\right) \\
\bar{e}_{R}=\bar{R}-R(\chi, \bar{I}) \\
\underline{e}_{R}=R(\chi, I)-\underline{R}
\end{array}\right.
$$

\section{Acknowledgements}

F. Mairet has been supported by Programa de Financiamiento Basal from the Center of Mathematical Modeling, Universidad de Chile, and by FONDECYT (Postdoctorado 3120117). O. Bernard benefited from the support of the Symbiose research project founded by the French National Research Agency (ANR) and from the Nautilus ARC, funded by INRIA.

\section{References}

[1] R.H. Wijffels and M.J. Barbosa. An Outlook on Microalgal Biofuels. Science, 329(5993):796-799, 2010.

[2] O. Bernard. Hurdles and challenges for modelling and control of microalgae for co2 mitigation and biofuel production. Journal of Process Control, 21:1378-1389, 2011.

[3] J.-L. Gouzé, A. Rapaport, and Z. Hadj-Sadok. Interval observers for uncertain biological systems. Ecological Modelling, 133:45-56, 2000.

[4] A. Rapaport and J.-L. Gouzé. Parallelotopic and practical observers for nonlinear uncertain systems. International Journal of Control, 76(3):237-251, 2003.

[5] H.L. Smith. Monotone dynamical systems: An introduction to the theory of competitive and cooperative systems. American Mathematical Society., 75, 1995. 
[6] O. Bernard and J.L. Gouzé. Closed loop observers bundle for uncertain biotechnological models. Journal of Process Control, 14:765-774, 2004.

[7] M. Moisan, O. Bernard, and J.-L. Gouzé. Near optimal interval observers bundle for uncertain bioreactors. Automatica, 45:291-295, 2009.

[8] G. Goffaux, A. Vande Wouwer, and O. Bernard. Improving continuous - discrete interval observers application to microalgae-based bioprocesses. Journal of Process Control, 19(7):1182-1190, 2009.

[9] N. Meslem, N. Ramdani, and Y. Candau. Using hybrid automata for set-membership state estimation with uncertain nonlinear continuous-time systems. Journal of Process Control, 20(4):481-489, 2010.

[10] F. Mazenc and O. Bernard. Interval observers for planar systems with complex poles. IEEE TAC, 55:523-527, 2010.

[11] M. Moisan and O. Bernard. Robust interval olservers for global lipschitz uncertain chaotic systems. Sys \& Ctrl Letters, 59:687-694, 2010.

[12] F. Mazenc and O. Bernard. Interval observers for linear time-invariant systems with disturbances. Automatica, 47:140-147, 2011.

[13] D. Efimov, L. Fridman, T. Raissi, A. Zolghadri, and R. Seydou. Interval estimation for lpv systems applying high order sliding mode techniques. Automatica, 48:2365-2371, 2012 .

[14] F. Mairet and O. Bernard. Coupling framers to get enhanced interval observers. application to growth rate estimation in a photobioreactor. In Proceedings of the 48th IEEE Conference on Decision and Control (CDC). Shangai, China, 2009.

[15] V. Lemesle and J.-L. Gouzé. Hybrid bounded error observers for uncertain bioreactor models. Bioprocess and Biosystems Engineering, 27:311-318, 2005.

[16] F. Mairet, M. Moisan, and O. Bernard. Interval observer with near optimal adaptation dynamics. application to the estimation of lipid quota in microalgae. International Journal of Robust and Nonlinear Control, 2013. To appear.

[17] R.J. Geider, H.L. MacIntyre, and T.M. Kana. A dynamic regulatory model of phytoplanktonic acclimation to light, nutrients, and temperature. Limnology and Oceanography, pages 679-694, 1998.

[18] K.J. Flynn. A mechanistic model for describing dynamic multi-nutrient, light, temperature interactions in phytoplankton. Journal of Plankton Research, 23(9):977, 2001.

[19] R.A. Armstrong. Optimality-based modeling of nitrogen allocation and photoacclimation in photosynthesis. Deep Sea Research Part II: Topical Studies in Oceanography, 53(5-7):513-531, 2006.

[20] F. Mairet, O. Bernard, P. Masci, T. Lacour, and A. Sciandra. Modelling neutral lipid production by the microalga Isochrysis aff. galbana under nitrogen limitation. Bioresource Technology, 102:142-149, 2011. 
[21] A. Packer, Y. Li, T. Andersen, Q. Hu, Y. Kuang, and M. Sommerfeld. Growth and neutral lipid synthesis in green microalgae: A mathematical model. Bioresource technology, 102(1):111-117, 2011.

[22] J. Quinn, L. De Winter, and T. Bradley. Microalgae bulk growth model with application to industrial scale systems. Bioresource technology, 102:5083-5092, 2011.

[23] F. Mairet, O. Bernard, T. Lacour, and A. Sciandra. Modelling microalgae growth in nitrogen limited photobiorector for estimating biomass, carbohydrate and neutral lipid productivities. In Proceedings of the 18th IFAC World Congress. Milano, Italy, 2011.

[24] M. R. Droop. Vitamin B12 and marine ecology. IV. the kinetics of uptake growth and inhibition in Monochrysis lutheri. J. Mar. Biol. Assoc., 48(3):689-733, 1968.

[25] M. R. Droop. 25 years of algal growth kinetics, a personal view. Botanica marina, 16:99-112, 1983.

[26] A. Sciandra and P. Ramani. The limitations of continuous cultures with low rates of medium renewal per cell. J. Exp. Mar. Biol. Ecol., 178:1-15, 1994.

[27] O. Bernard and J.-L. Gouzé. Nonlinear qualitative signal processing for biological systems: application to the algal growth in bioreactors. Math. Biosciences, 157:357$372,1999$.

[28] B.P. Han. Photosynthesis-irradiance response at physiological level: a mechanistic model. Journal of theoretical biology, 213(2):121-127, 2001.

[29] PHC Eilers and JCH Peeters. Dynamic behaviour of a model for photosynthesis and photoinhibition. Ecological modelling, 69(1-2):113-133, 1993.

[30] O.N. Ross and R.J. Geider. New cell-based model of photosynthesis and photoacclimation: accumulation and mobilisation of energy reserves in phytoplankton. Marine Ecology Progress Series, 383:53-71, 2009.

[31] F. Mairet, M. Titica, O. Bernard, and J. Pruvost. Coupling biological and radiative models to describe microalgal growth in a photobioreactor. In Proceedings of the 11th Computer Applications in Biotechnology (CAB),. Leuven, Belgium, 2010.

[32] T. Lacour, A. Sciandra, A. Talec, P. Mayzaud, and O. Bernard. Neutral lipid and carbohydrate productivities as a response to nitrogen status in Isochrysis sp.(t-iso; haptophyceae): starvation versus limitation. Journal of Phycology, 48:647-656, 2012.

[33] T. Lacour, A. Sciandra, A. Talec, P. Mayzaud, and O. Bernard. Diel variations of carbohydrates and neutral lipids in nitrogen-sufficient and nitrogen-starved cyclostat cultures of Isochrysis sp. Journal of Phycology, 48:966-975, 2012. 